\title{
Kirchliche Kommunikation der Zukunft
}

\author{
von Andreas-Maria Deskur
}

Wissenschaftlich fundierte Vorhersagen lassen sich schwer machen, vor allem, wenn es sich um Dinge von allgemeiner und umfassender Tragweite handelt, die gleichzeitig vom Verlauf der Geschichte, vom technischen Fortschritt, von der kulturellen Entwicklung, der menschlichen Freiheit und den unerforschlichen Ratschlïssen Gottes abhängen.

Wird es ein Jahr 2000 geben? Wir können das nicht mit Sicherheit sagen. Der Planet Erde, ein Staubkorn im unendlichen Weltall, ist sehr vielen mutmaßlichen oder noch unbekannten Gefahren ausgesetzt. Die Offenbarung sagt, daß das Ende „wie ein Dieb“ kommen wird, wenn wir es am wenigsten erwarten.

Trotzdem müssen wir Menschen, und vor allem wir Christen, so leben, arbeiten, planen, projektieren, als müßte nicht nur das Jahr 2000 kommen, sondern noch viele weitere Jahre.

Der Versuch ist dann so phantastisch nicht, vorherzusehen, was im Jahr 2000 geschehen wird, oder besser, was geschehen müßte, wenigstens soweit das den Beitrag der Kirche betrifft. Wir sollten dabei nicht vergessen, daß die Kinder, die wir jetzt auf die Erstbeichte und Erstkommunion vorbereiten, im Jahre 2000 dreißig Jahre alt werden, zur Reife, zur Fülle ihrer persönlichen Entwicklung und ihrer gesellschaftlichen Leistung kommen, während die Erwachsenen von heute in ihrer Mehrzahl bei Beginn des dritten Jahrtausends noch einen bestimmenden Einfluß ausüben.

\section{Der technische Gesichtspunkt}

Die soziale Kommunikation in dem Sinn, den das Zweite Vatikanische Konzil diesem Begriff gegeben hat, ist durch den technischen, vor allem elektronischen Fortschritt möglich geworden und bleibt in ihrer Entwicklung und in gewisser Weise auch in ihren Möglichkeiten von diesem bedingt und bestimmt. Auf diesem Gebiet können wir uns jetzt, mit großer Wahrscheinlichkeit und ohne Jules Verne zu spielen, im Jahr 2000 einen Menschen vorstellen, der zu Hause seine Tageszeitung aus der Transistorbox zieht, der eine Armbanduhr trägt, auf deren Bildschirm er zahlreiche, mit Simultanübersetzungen ausgestattete Farbfernsehprogramme verfolgen kann; der jedes Schauspiel, jedes Konzert, jedes Ereignis, sowie sie auf nationaler oder internationaler Ebene in den Informationsspeichern „im Bild“ sind, auf seinem häuslichen Bildschirm reproduzieren kann; der im Vorführraum dreidimensionalen und stereofonen Projektionen beiwohnt, die ihm die uneingeschränkte Illusion geben, selber an den Ereignissen beteiligt zu sein.

Bischof Andreas-Maria Deskur ist Präsident der Päpstlichen Kommission für die Sozialen Kommunikationsmittel, Vatikanstadt. 


\section{Wesentlicher Bestandteil der Kultur}

Im Jahr 2000 wird der Mensch einen immer größeren Teil seiner Zeit der Kommunikation widmen. Sie wird dann ein Viertel, vielleicht sogar ein Drittel seines Lebens ausmachen. Selbst die Zeit zum Schlafen und Arbeiten kann von der Kommunikation, wenigstens zum Teil, beherrscht sein.

Die menschliche Kreativität auf künstlerischem, kulturellem, wissenschaftlichem, sozialem und politischem Gebiet wird der beherrschenden Rolle der sofortigen Kommunikation Rechnung tragen müssen. Sie wird sich also von vornherein in der Perspektive der sozialen Kommunikation verstehen, abwickeln und darstellen.

Die infolge einer verfeinerten Technik und des natürlichen Wachstums der BevöIkerung vermehrten sozialen Beziehungen werden die Strukturierung der Gesellschaft immer komplexer machen, die, um regiert zu werden, sich zu entwickeln und $\mathrm{zu}$ entfalten, auf ständig fortgeschrittenere Formen der Kommunikation zurüdkgreifen muß.

In diesem Kontext wird sich auch das religiöse Leben des Menschen entwickeln; auch die Verkündigung der christlichen Botschaft und das Leben des Gottesvolkes, das die Kirche ist, werden diesen Entwicklungsgang nehmen.

Wie in der menschlichen Gesellschaft, wird auch in der Gemeinschaft der Gläubigen mit ihrer göttlich verfaßten Ordnung, ihrem Lehramt und ihrem sakramentalen Dienst das Leben einer Ortsgemeinde - in der der einzelne seine Identität wahrt, seine spezifischen kulturellen Werte in Leben umsetzt und sie mit den anderen Mitgliedern dieser Gemeinde austauscht, die er persönlich kennenlernen kann - dank der Entwicklung der sozialen Kommunikation gleichzeitig eine bereichernde Steigerung erfahren, nämlich durch die Teilhabe am Leben des "Welt-Dorfs“, wo die Menschheit die Fülle ihrer Werte, ihrer Reichtümer und ihrer Möglichkeiten in Leben umzusetzen sucht und die Kirche ihre Raum und Zeit übergreifende Universalität verwirklicht. Noch mehr, der Wunsch nach Teilnahme durch Kommunikation an allen Reichtümern der Vergangenheit, nach innerer Verbundenheit mit den Menschen nicht nur aller Rassen und Herkunft, sondern auch aller Zeiten, wird sich stärker bemerkbar machen. Damit wird in der Menschheit stärker das Spirituelle, das Unvergängliche gespürt, also das Spiegelbild des Ewigen, das Bild Gottes, das der Mensch von Natur aus in sich trägt - mit der daraus resultierenden geheimnisvollen Erwartung eines persönlichen Kontaktes mit ihrem unsichtbaren Vater.

\section{Verteidigung der bedrohten Freibeiten}

Die Entwicklung der sozialen Strukturen, das Wachstum der Bevölkerung und der technische Fortschritt bieten dem Menschen persönlich enorme neue Vorteile, aber sie verlangen auch neue Großherzigkeit, neue Opfer.

Indem die soziale Kommunikation ein bestimmender Teil des täglichen Lebens wird, verlegt sie die ideologischen und politischen Auseinandersetzungen viel mehr als bisher in das Bewußtsein jedes einzelnen. Wie in der Vergangenheit Regimenter und Kanonen, so werden immer mehr die Instrumente der sozialen Kommunikation zu bestimmten Zwecken benutzt. Daher die wachsende Gefahr, daß jede Gesellschaft, die nach Herrschaft über nahe und ferne Gebiete strebt, jede politische Partei, jede ideologische Gruppe sich dieser Mittel, als wären sie Basen für interkontinentale Raketen, zu bemächtigen sucht, indem sie mit stärkerer Macht, wenn nicht sogar aus- 
schließlich, ihren Druck auf den Gebrauch dieser Mittel ausübt, um von deren wachsender Fähigkeit, die Weisen des Sehens, Wollens und Denkens zu beeinflussen, zu profitieren.

Die Sicherung des freien Zugangs zu den Medien, der Objektivität und Unparteilichkeit der Kommunikatoren, des Rechtes auf Erwiderung und Verteidigung dessen, der politisch oder wirtschaftlich schwach, auf der Ebene der geistlichen und kulturellen Werte aber vielleicht reich ist, wird eines der Hauptprobleme des Gleichgewichts und des menschlichen Fortschritts im Jahr 2000 sein.

Die Kirche ist in der gegenwärtigen Menschheit vielleicht der wichtigste Hilfsfaktor für die Verteidigung der Freiheit des Kommunikators. Das Zweite Vatikanische Konzil hat in seinem Dekret "Inter mirifica“ die Notwendigkeit dieser neuen Aufgabe klar gesehen (Art. 5 und 12). In der Tat läßt sich die menschliche Freiheit nicht garantieren ohne einen tiefen Glauben an die Würde des Menschen, und dieser Glaube kann keine festere Grundlage haben als die Botschaft von der Menschwerdung!

Wie die Erfahrung andererseits lehrt, stellt die Freiheit der religiösen Kommunikation ein verläßliches Barometer der Freiheit der Kommunikation überhaupt dar. Soll die Kommunikationsfreiheit eingeschränkt werden, werden als erste die religiöse Presse und religiöse Sendungen unterdrückt, und sie werden erst als letzte wieder zugelassen, wenn man zu dieser so grundlegenden und unverzichtbaren Freiheit zurückkehren will.

Aber die Freiheit des Kommunikators reicht noch nicht hin, die Freiheit der sozialen Kommunikation sicherzustellen. Die letztere hängt auch von der Freiheit des Empfängers ab, von der Freiheit zu wählen, von der Freiheit sich zu entscheiden, von der Freiheit zu einem reifen Urteil, schließlich von der Freiheit, durch die soziale Kommunikation unterstiitzt und bereichert, nicht aber unterjocht, ferngeleitet und manipuliert zu werden.

Weil es sich um ein tiefes Erziehungsproblem handelt, bei dem hohe Ideale, sittliche Werte, persönliche Entscheidungen und das ewige Schicksal des Menschen zum Einsatz kommen, kann keine Gesellschaft, auch nicht die des Jahres 2000, ihr Gleichgewicht wahren, ohne daß der einzelne, wenn er sein Leben persönlich verantworten will, sich auf religiöse Werte, ja auf die absolute Gewißheit dieser Werte stützen und sich ein hohes, wenn auch schwer zu erreichendes Lebensideal vor Augen halten kann. Da sieht sich die Kirche auf dem Gebiet der Kommunikation vor eine neue, harte, aber unersetzlich wichtige Aufgabe gestellt, nämlich jedem Menschen die Gewißheit der Werte, eine klare Sicht seiner Würde und den Sinn seines Lebens zu vermitteln und ihn mit jenen übernatürlichen Kräften auszurüsten, die er braucht, um ein hohes Lebensideal anzustreben und auch zu verwirklichen, ein Ideal, das ihm erlaubt, aus der Informationsflut der sozialen Kommunikation aufzutauchen und so daraus eher Gewinn zu schöpfen wie aus reinem Wasser und echter Nahrung, und dies im Verlauf seines ganzen Lebens, für das er immer verantwortlich und über das er Herr bleiben muß, auch wenn er nur einer von 5 oder 10 Milliarden Bewohnern der Erde ist.

\section{Notwendigkeit geistiger Ökologie}

Sehr spät, wir hoffen nicht zu spät, hat die Menschheit - haben die Verantwortlichen im gesellschaftlichen und öffentlichen Leben -- dem Rechnung getragen, daß 
der Fortschritt auch Schlacken abwirft, daß neben den Früchten der Urbanisierung, der industriellen Entwicklung und der Gewinnung neuer Energiequellen sich Haufen von Abfällen und Giftstoffen bilden und die Verschmutzung der Umwelt sich auszubreiten beginnt, wodurch die Reichtümer, die Gott dem Menschen mit der Natur und ihren Gesetzen gegeben hat, vernichtet und geschändet werden. Die zunehmende Fülle, ja die Explosion der Kommunikationen beginnt bereits, auf menschlicher Ebene solche Schlacken abzuwerfen und Verschmutzung hervorzurufen. Nur gibt man sich noch nicht voll Rechenschaft darüber, so groß ist die Euphorie über die freie und oft ungeordnete Verbreitung von Nachrichten, Film- und Funkproduktionen, Ideen, Verhaltensweisen und Provokationen. Die menschlidhe Natur, ihr biologisches, psychosomatisches und geistiges Leben, hat ihre Gesetze, die zur gleichen Zeit Schutzwehr und Führer sind, Garanten des Glücks und der kostbarsten Güter: einer selbstlosen Liebe, der Selbstbeherrschung, der Ehrfurcht vor der Wahrheit, des Dankes für die empfangenen Güter, des Verlangens, Gutes zu tun, der Hoffnung, für immer die Beziehungen von Person zu Person bewahren zu können, die nicht der Vergänglichkeit der Materie unterworfen sein wollen.

Die ganze Skala der Tugenden, die zur menschlichen Natur gehören, die den Reichtum der vergangenen Kulturen einschließen und zusammenfallen im Begriff der „Moral“, könnten, wie die von den industriellen Abwässern verschmutzte Natur, angesichts der gigantischen Flut der Kommunikationen im Jahr 2000 in Gefahr sein. Wer könnte einen wirksameren Reinigungsfilter bieten, damit diese Flut trinkbar wird, wenn nicht die christliche Gewissensbildung? Wer könnte der gesamten Gesellschaft wie den einzelnen Gemeinschaften des Jahres 2000 weisere und wirksamere Hilfsmittel vorschlagen als gerade die Kirche, die es - in der alten und noch mehr in der modernen Welt - praktisch allein verstanden hat, Verfolgungen und Spott ebenso zu trotzen wie der Unpopularität, weil sie sich widerstandsfähig zeigte

gegenüber modischen Ideen, entfesselten Leidenschaften, der Verschwörung wirtschaftlicher und politischer Interessen, immer dann, wenn eine Wahrheit, eine Tugend, ein Wert, eine Seite der menschlichen Würde bedroht, geschmälert oder mit Füßen getreten wurde?

Es stimmt, daß viele Religionen, wie das Zweite Vatikanische Konzil betont, in der Vergangenheit zur Erhaltung der kostbarsten sittlichen, persönlichen und familiären Werte beigetragen haben und, wie wir hoffen, auch in Zukunft beitragen werden. Aber das Problem der geistigen Okologie besteht in der Notwendigkeit, wirklich alle diese Werte zu erhalten, und um das tun zu können, muß es Hüter der Gewißheit für diese Werte geben, die für das ganze menschliche Leben von Bedeutung sind. Oder stimmt es nicht, daß ein Element genügt, um das gesamte ökologische Gleichgewicht zu stören?

Angesichts der bis zum Jahre 2000 noch wachsenden und oft nicht ungetrübten Flut der Kommunikationen wird der wichtigste Beitrag der Kirche darin bestehen, dem Menschen, jedem Menschen an jedem Tag, auf geistig-religiöser Ebene ein Glas reinsten Wassers zu reichen.

\section{Kreativität}

Diese Überlegungen könnten allzu negativ scheinen - auch wenn im christlichen Denken das Negative seine ganze Kraft aus den positiven Werten zieht, die es ver- 
teidigen muß -, würden wir nicht auch von dem Beitrag sprechen, den die Welt des Jahres 2000 von der Kirche in einem wichtigen Bereich der sozialen Kommunikation erwarten darf: dem der Kreativität. Die Kommunikationsinstrumente fressen im wahrsten Sinn des Wortes Informationen, Programme, spektakuläre Ereignisse, wobei sie dem Neuen, dem nie Gesehenen und nie Gehörten den Vorzug geben, dem, was sich leicht erfassen, was sich gefällig aneignen läßt. Sie erlauben nicht bloß Sehen und Hören, sondern setzen den ganzen Mechanismus des menschlichen Denkens und Fühlens in Bewegung. Dabei sind sie der dauernden Versuchung ausgesetzt, bei der Kommunikation zu "schwindeln“, indem sie Surrogate als wahr, interessant und nützlich anbieten oder vergessen lassen, daß „nicht alles Gold ist, was glänzt“, oder der Eile die Wahrhaftigkeit und Gründlichkeit in der Darstellung opfern und die Grenze zwischen dem Dargestellten und der Wirklichkeit, zwischen der Fiktion und dem Leben verschwinden lassen.

Die außerordentlichen Möglichkeiten der einzelnen Kommunikationstechniken sind nicht nur zum Teil unerforscht geblieben und in ihrer Wirkung nicht ausreichend erkannt, sondern vor allem noch nicht genutzt, um den örtlichen und nationalen Gemeinschaften und der ganzen Menschheitsfamilie den ganzen Reichtum an Austausch, Informationen, Ideen und Künsten zu vermitteln, mit dem die Menschheit ihre eigene Kreativität steigern und ihre besten und uneigennützigsten Talente in den Dienst der sozialen Kommunikation stellen könnte.

Die Kreativität des Menschen zu fördern und sie - unter dem Gesichtspunkt der sozialen Kommunikation - wirksam in den Dienst der wahren Werte zu stellen, speziell derer, die die Gesamtentwicklung des Menschen, seine geistliche und ewige Dimension betreffen, wird immer eine Aufgabe bleiben, die die Möglichkeiten der irdischen Autoritäten bei weitem überschreitet.

Die Kirche muß auf diesem Gebiet jene anspornende Rolle übernehmen, die es demjenigen, der mit den entsprechenden Talenten ausgestattet ist, ermöglicht, die wahren Werte zu entdecken und in die Sprache der sozialen Kommunikation zu übersetzen, indem er ein Bild der Geschichte, der Kultur und der Religion zeichnet, das ihrer Bedeutung für die Menschenfamilie angemessen ist.

Wenn wir heute die Entsakralisierung unserer Zeit beklagen - in dem Sinn, daß der Bezug des persönlichen, familiären und bürgerlichen Lebens zum Gespräch zwischen Gott und dem Menschen verloren ist, zwischen Gott als dem liebenden Erlöser und dem Menschen, der zwar ein aufsässiger Sohn ist, aber im Grunde fähig bleibt, seinem himmlischen Vater zu gehorchen und dessen Vollkommenheit widerzuspiegeln -, dann tun wir das, weil in der Kreativität der sozialen Kommunikation, zumindest zum Teil, der gerade den Christen eigentümliche Beitrag fehlt, das Geheimnis der Menschwerdung mit all seinen Konsequenzen in die Sprache der modernen Mittel der sozialen Kommunikation verständlich, wirksam und doch getreu zu übersetzen.

Der vom Zweiten Vatikanischen Konzil vorgezeichnete und von Papst Paul VI. in der Pastoralinstruktion "Communio et progressio" wiederaufgenommene Weg bietet die Hoffnung, daß die gesamte Kirche sich im Jahr 2000 auf diese Aufgabe einer neuen Kreativität vorbereitet findet. 


\section{S UM M A R Y}

Technical developments in Communications will continue in the year 2000. The time taken up by communications will be between a third and a quarter of human life. There will be the growing desire to participate in the riches of the past as well as in those of men of all races and all times. Social Communications will be a decisive part of daily life. Therefore discussions on ideologies and politics will occupy a larger part in the life of the individual than formerly. Just as in the past armies and guns have been instruments for certain purposes, communications media will then replace them. It is here that the Church has to defend the real freedom of both communicator and recipient. The Church will have to give a clear view of the dignity and meaning of human life. The communications explosion also creates pollution in human society. Here the promotion of the Christian conscience has to become the filter for unpolluted communications. But the Church also has to contribute towards human creativity. She must help the different communication techniques to foster the creativity of man and of human society. The mystery of the Incarnation has to be realized in the language of modern communications media in a way that is understandable, effective and true.

\section{RÉS U MÉ}

Les progrès techniques vont se poursuivre et le temps de la communication déterminera, en l'an 2000, un quart ou même un tiers de la vie humaine. Le souhait à la participation par la communication à toutes les richesses du passé, le souhait au rattachement intérieur avec des hommes de toutes les rasses, de toutes les origines et de tous les temps sera visiblement plus fort. Par le fait que la communication sociale devient une partie déterminante de notre vie de tous les jours, elle déplace aussi les démélés idéologiques et politiques plus qu'autrefois dans la conscience de chacun en particulier. De même que les régiments et les canons dans le passé, ainsi les instruments de la communication sociale seront utilisés à des fins bien déterminées. Ici, l'Eglise a à défendre la véritable liberté; pour cela, à la liberté du communicateur doit correspondre celle du receveur; l'Eglise doit donner une vue claire de la dignité et du sens de la vie humaine. Egalement, l'explosion de la communication commence, sur le plan humain, à jeter des impuretés et à faire naître des saletés. La formation de conscience chrétienne doit offrir ici un filtre de purification. Mais alors l'Eglise doit aussi collaborer à la créativité. Elle doit aider pour que chaque technique de communication en particulier encourage la créativité de l'homme et de la communauté humaine. Le secret de l'incarnation doit être traduit de façon intelligible, efficace et cependant fidèle dans la langue des moyens de communication modernes.

\section{RES UMEN}

Proseguirá el desarrollo técnico y la era de la comunicación social condicionará en el año 2000 una cuarta parte 6 hasta un tercio de la vida humana. Se manifestará más el deseo de participar mediante la comunicación social en todos los tesoros del pasado, en la vinculación interior con los hombres de todas las razas, de todos los orígenes y de todos los tiempos. A medida que la comunicación social se convierte en parte de la vida cotidiana, profundizan también en la consciencia de cada individuo, más que hasta el presente, las controversias ideológicas y políticas. Del mismo modo que en el pasado se utilizaron regimientos y cañones, se recurrirá en el futuro a los instrumentos de la comunicación social. En este contexto corresponde a la Iglesia defender la verdadera libertad; la lifertad del comunicante debe respetar la del receptor; la Iglesia debe en este punto difundir una visión clara de la dignidad y sentido de la vida humana. También la explosión de medios de difusión comienza a producir resíduos y contaminación. La formación de la conciencia cristiana puede ofrecer un filtro. La Iglesia tiene además que aportar uno contribución creadora. Debe ayudar a que la técnica de la comunicación individual promocione la creatividad del hombre y de la sociedad humana. El misterio de la Encarnación debe traducirse al lenguaje de los modernos medios de comunicación de forma comprensible, atractiva y, sin embargo, fiel. 